Voix et Images

voixetimages

\title{
La valse des amoureux
}

\section{Lori Saint-Martin}

Volume 19, numéro 2 (56), hiver 1994

\section{Anne-Marie Alonzo}

URI : https://id.erudit.org/iderudit/201102ar

DOI : https://doi.org/10.7202/201102ar

Aller au sommaire du numéro

\section{Éditeur(s)}

Université du Québec à Montréal

\section{ISSN}

0318-9201 (imprimé)

1705-933X (numérique)

Découvrir la revue

Citer cet article

Saint-Martin, L. (1994). La valse des amoureux. Voix et Images, 19(2), 412-416.

https://doi.org/10.7202/201102ar d'utilisation que vous pouvez consulter en ligne.

https://apropos.erudit.org/fr/usagers/politique-dutilisation/ 
Féminismes

\section{La valse des amoureux}

\section{Lori Saint-Martin, Université du Québec à Montréal}

Il arrive, le temps d'un best-seller, qu'un effet de mode fasse croire à un mouvement de société. Cette année, se multiplient les livres de réflexions sur le couple: essais à une voix, chez Denise Bombardier, ou à deux, chez Sylvie Chaput et Marc Chabot. Qu'il y ait engouement pour la question, les chiffres de vente le disent assez. Pourtant, le sujet, usé jusqu'à la corde - les innombrables livres de psychologie populaire américaine y ont vu -, est des plus casse-cou: on risque toujours, comme l'ont fait en France Bernard-Henri Lévy et Françoise Giroud ${ }^{1}$, de débiter d'aimables banalités, ou, mâtinées de force références romanesques, des vérités si générales qu'elles ne valent plus d'être dites.

Le retour en force du couple (hétérosexuel, il va sans dire): signe d'un nouveau conservatisme social, d'un repli sur les valeurs sûres du foyer? Ou déplacement de la réflexion féministe vers des enjeux qui, à défaut d'être nouveaux, préoccupent un très grand nombre de femmes? À voir. Et qu'y trouvent les lectrices (on peut les supposer majoritaires) et les lecteurs? Pour peu qu'on lise les deux essais, on ne saura plus où donner de la tête. Car nos auteurs en arrivent à des 
conclusions opposées. À l'optimisme relatif de Sylvie Chaput et Marc Chabot appelant "la fin du combat "s'oppose la "déroute des sexes" de Denise Bombardier. Qui a raison? Allons voir de plus près.

Le couple Chaput-Chabot a eu l'excellente idée de présenter une réflexion à deux sur les relations homme-femme. Voilà enfin un procédé original, à condition d'aller au fond des choses. À y regarder de plus près, toutefois, on se rend compte que chacun a pensé et écrit de son côté, sur des questions différentes, et qu'on n'a fait, ensuite, que juxtaposer les textes.

Marc Chabot en appelle à une véritable "pensée hétérosexuelle", une pensée qui vise "le dépassement des sexes, comme le dépassement de l'enfermement dans la masculinité ou dans la féminité ${ }^{2}$. Contre le retranchement, contre l'affrontement stérile, il cherche, appelle la femme: "c'est avec l'autre que je peux penser la vie" (p. 151). Si les moyens d'y parvenir demeurent vagues - comment éviter que le dépassement signifie la disparition du féminin, comme on l'a vu si souvent? - , le rapprochement qu'il évoque séduit et fait rêver.

Vifs et combatifs, les trois essais de Sylvie Chaput s'attaquent à des cibles masculines (on est dans la polémique féministe - très bien menée d'ailleurs - plutôt que dans la mixité souhaitée par son compagnon): la contraception dure qui n'a pas rempli ses promesses; Père manquant, fils manqué de Guy Corneau, qui accable les mères trop présentes et fait du seul père le créateur potentiel de l'homme nouveau; la lettre de Marc Lépine, l'homme qui a tué "les femmes avec lesquelles il aurait dû vivre" (p. 167).

Deux registres, donc, deux solitudes de la pensée. Les textes de l'un ne rejoignent d'aucune façon les textes de l'autre. Certes, écrire ensemble aurait entraîné un nivellement des idées comme du langage. Comment, d'ailleurs, en arriver à vraiment penser à deux? (Pas si évident, le "dépassement des sexes „...) Peut-être pour cela aurait-il fallu que chacun traite, de son côté, le même sujet (que pense Marc Chabot du livre de Guy Corneau, par exemple?) et que les deux textes se suivent. En l'état actuel des choses, pas de véritable mixité de la pensée; il s'agit plutôt de deux réflexions parallèles, toutes deux intéressantes, mais qui ne gagnent rien à être réunies.

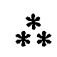

"Les généralisations sont devenues impossibles, voire impensables", écrit Marc Chabot (p. 84). Denise Bombardier n'est pas de son avis: à 
mi-chemin entre la réflexion et le "how-to" à l'américaine, La Déroute des sexes ${ }^{3}$ en regorge. L'auteure ne cesse d'affirmer, sur tous les tons, que les "nouvelles femmes" resteront sur le carreau si elles ne changent pas, et vite. Sa thèse est très simple, et quelque peu janséniste: le féminisme a entraîné pour les femmes des améliorations sur le plan économique, mais au prix du bonheur dans les relations amoureuses. (Quant à savoir comment se mesure le bonheur - les femmes de ce mythique "avant "nageaient-elles vraiment dans la félicité? -, le texte est muet). La menace est claire: "les "nouvelles femmes" représentent un défi que peu d'hommes veulent relever" (p. 19). Et voilà donc relancée la guerre entre les femmes: guerre des "féministes" contre les "traditionnelles", toutes rivales à la poursuite de l'homme, toutes en quête du couple stable, promu idéal universel et apogée du bonheur.

"Il y a un prix à payer pour accéder à l'égalité des sexes", affirme Denise Bombardier (p. 19). Sans aucun doute - máis il y a aussi un prix à payer pour ne pas y accéder. Certains de ses arguments - le féminisme tue l'amour ${ }^{4}$, toute tentative de brouiller les distinctions entre les sexes sème le désordre - se trouvaient déjà chez les antiféministes du siècle dernier ${ }^{5}$. Remis au goût du jour, les voilà de nouveau en circulation. S'y ajoute un éloge de la passion, l'élément le plus touchant du livre, et un appel à la réconciliation des sexes qui sonne juste. Ce sont les moyens d'y parvenir qui ne feront pas l'unanimité.

Certes, on vit une époque de bouleversement dans les relations entre les sexes; tout a basculé, et les femmes autant que les hommes ressentent parfois la nostalgie de ce bon vieux temps (mythique, bien sûr) où "tout était simple". Mais Denise Bombardier fait totalement abstraction des relations de pouvoir et, du coup, voit les femmes comme les uniques coupables des souffrances des deux sexes: "Peuvent-elles rendre les hommes responsables de leurs malheurs, eux qui n'ont ni imaginé ni souhaité les changements dont elles ont dû être les initiatrices?» (p. 10). Le désarroi des hommes existe bel et bien, personne ne songerait à le nier. Il est d'ailleurs assez piquant qu'une femme se fasse leur championne. Mais de là à approuver en bloc leur conduite - ils ont raison au fond d'avoir du mal à aimer une femme qui réussit, raison d'aspirer au repos du guerrier -, il y a un pas. En revanche, l'auteure frappe juste lorsqu'elle souligne des contradictions: pourquoi certaines femmes se transforment-elles en hommes (en privilégiant le travail au détriment de l'amour, par exemple) tout en exigeant de leurs compagnons une tendresse nouvelle? Tout cela, Denise Bombardier n'est pas la première à le dire — on trouve ici plus d'une lapalissade —, mais certaines vérités méritent d'être rappelées. 
Pour avancer, "il faudra surtout reprendre l'éducation des sexes sur des bases complètement nouvelles" (p. 75), écrit Denise Bombardier. Contradiction? Elle semble en appeler à une évolution qu'elle reproche pourtant aux "battantes" de vouloir provoquer. Est-il souhaitable ou non que l'homme se transforme? S'il est vrai que la plupart auraient du mal à aimer une femme qui ne vit pas dans leur ombre, comme l'affirme Denise Bombardier, faut-il revenir à l'alternative traditionnelle: ou bien la carrière, ou bien le mari ? À elles de changer, une fois de plus: abolir leurs contradictions (comme si l'ambivalence n'était pas au coeur de chaque être humain), rassurer les hommes en mal de certitudes, réinvestir la famille. Rassurés, ils ne fuiront plus, nous promet l'auteure. Car "dans la chambre à coucher, on ne négocie pas" (p. 20). Drôle de vision de l'amour comme parfaitement pur, innocent, non contaminé par des rapports de force...

Tout aussi gênant, le discours sur le féminisme (on se trouve, selon l'expression plus que discutable de l'auteure, dans "l'aprèsféminisme "). Les qualificatifs délirants: "panzer de la guerre des sexes " (p. 29), "ces conquérantes" (p. 61), "ces amazones" (p. 66), "les troupes de choc de l'armée des amoureuses" (p. 73), "ces machos au féminin" (p. 81), "les guerrières de la lutte des sexes" (p. 88), "ces reines de la libération" (p. 124), rappellent curieusement la rhétorique antiféministe d'un Henri Bourassa:

Lorsque la femme émancipée aura bien battu la poussière du forum et clapoté dans la boue du fossé, elle viendra chez nous rapprendre de nos femmes ignorantes la saine notion de la maternité et de la véritable dignité féminine ${ }^{6}$.

Comme tous les discours révolutionnaires, le discours féministe a eu ses excès et ses ratés, il convient de le rappeler. Mais il est malaisé de dénoncer un mouvement dont on a tant profité soi-même. "Je voulais tout: amour, carrière, enfant " et "J'ai eu tout cela" (p. 8), avoue Denise Bombardier. Pourtant elle conspue - au nom de quelle morale, au juste? - celles qui "revendiquent l'orgasme ET l'égalité salariale" (p. 16), et, sévère, les somme de "cesser de tout vouloir à la fois" (p. 135). Acte de contrition d'une femme célèbre qui déconseille la gloire aux autres? La pirouette étonne.

La réconciliation des hommes et des femmes, la recherche de nouvelles formes d'amour, tout le monde les souhaite. Si l'essai de Denise Bombardier y contribue, tant mieux. Pour ma part, j'en doute. Un retour - réel ou symbolique - des femmes au foyer n'est plus possible. C'est sur d'autres bases, encore à inventer, que devra s'établir le nouveau dialogue entre les sexes. 
1. Bernard-Henri Lévy et Françoise Giroud, Les Hommes et les Femmes, Paris, Olivier Orban, 1993.

2. Sylvie Chaput et Marc Chabot, À nous deux! Hommes et femmes: la fin du combat?, Montréal, Le Jour/VLB éditeur, 1993, p. 30.

3. Denise Bombardier, La Déroute des sexes, Paris, Seuil, 1993, 138 p.

4. Et pourtant, dit-elle, les femmes les plus amères, de nos jours, ne sont pas les pionnières du féminisme, mais *ces femmes qui dénonçaient, il y a vingt ans, la cause féministe en général et les féministes en particulier * (p. 30).

5. Voir entre autres Mireille Dottin-Orsini, Cette femme qu'ils disent fatale. Textes et images de la misogynie fin-de-siècle, Paris, Grasset, 1993.

6. Henri Bourassa, Femmes-hommes ou hommes et femmes? Études à bâtons rompus sur le féminisme, Montréal, Imprimerie du Devoir, 1925, p. 18. 